\title{
Flat Twistor Spaces, Conformally Flat Manifolds and Four-Dimensional Field Theory
}

\author{
M. A. Singer \\ Mathematical Institute, Oxford OX1 3LB, United Kingdom \\ Received July 20, 1989; in revised form February 27, 1990
}

\begin{abstract}
A definition is proposed of "four-dimensional conformal field theory" in which the Riemann surfaces of two-dimensional CFT are replaced by (Riemannian) conformally flat four-manifolds and the holomorphic functions are replaced by solutions of the Dirac equation. The definition is investigated from the point of view of twistor theory, allowing methods from complex analysis to be employed. The paper fills in the main mathematical details omitted from the preliminary announcement [15].
\end{abstract}

\section{Introduction}

In [23], Segal gives axioms for the notion of two-dimensional conformal field theory (CFT): the basic components are the (conformal equivalence classes of) compact Riemann surfaces $\Sigma$ with parametrized boundary, together with the natural operations of disjoint union

$$
\left(\Sigma_{1}, \Sigma_{2}\right) \mapsto \Sigma_{1} \bigsqcup \Sigma_{2},
$$

and contraction

$$
\Sigma \mapsto \Sigma^{\prime},
$$

where $\Sigma^{\prime}$ is obtained from $\Sigma$ by using the parametrization to attach a pair of boundary circles to each other.

A two-dimensional conformal field theory is then defined as a "Hilbert-space representation" of (1.1) and (1.2), that is a functor $\rho$ with the following properties: there is a Hilbert space $\mathscr{H}$ with

$$
\rho\left(S^{1}\right)=\mathscr{H}
$$

and if $\Sigma$ has $p$ positively oriented and $q$ negatively oriented boundary circles then

$$
\rho(\Sigma): \mathscr{H}^{\otimes p} \otimes \overline{\mathscr{H}}^{\otimes q} \rightarrow \mathbb{C}
$$

is a trace-class multilinear functional which satisfies

$$
\rho\left(\Sigma_{1} \bigsqcup \Sigma_{2}\right)=\rho\left(\Sigma_{1}\right) \otimes \rho\left(\Sigma_{2}\right)
$$


and

$$
\rho\left(\Sigma^{\prime}\right)=\operatorname{trace} \rho(\Sigma)
$$

(where "trace" is the "contraction map" induced by the inner product $\mathscr{H} \otimes \overline{\mathscr{H}} \rightarrow \mathbb{C}$ applied to the relevant factors $\mathscr{H}$ and $\overline{\mathscr{H}}$ ).

In the basic example, the "classical fields" are two-dimensional spinor fields, i.e. holomorphic sections of $\kappa^{1 / 2}$, the square root of the canonical bundle. The "classical phase space" is the space $H$ of $L^{2}$ spinors on the circle equipped with the standard polarization $H=H_{+} \oplus H_{-}$, where $H_{ \pm}$is the subspace of spinors that admit holomorphic continuations into the northern/southern hemisphere.

The polarization determines a (restricted) Grassmannian $\operatorname{Gr}(H)$ [20] which consists of those closed linear subspaces $W \subset H$ for which the orthogonal projection

$$
\mathrm{pr}_{+}: W \rightarrow H_{+} \text {is Fredholm }
$$

and the orthogonal projection

$$
\mathrm{pr}_{-}: W \rightarrow H_{-} \text {is trace-class. }
$$

In $[20$, Sects. 10.1-10.2] it is shown that the Fermionic Fock space $\mathscr{H}=\hat{\Lambda}\left(H_{+} \oplus \bar{H}_{-}\right)$lies between the space $\Gamma$ of holomorphic sections of Det*, the dual of the determinant line bundle on $\mathrm{Gr}$, and its antidual $\bar{\Gamma}^{*}$,

$$
\bar{\Gamma}^{*} \subset \mathscr{H} \subset \Gamma,
$$

both inclusions being dense. It follows that any point $W \in \mathrm{Gr}$ determines a functional

$$
\operatorname{eval}_{W}: \mathscr{H} \rightarrow \operatorname{Det}_{W}^{*}
$$

by evaluation of holomorphic sections at $W$. (This is a geometric formulation of the Bogolubov transformation by which a change in polarization of the classical phase space determines a new vacuum state.) The relevance of this theory to the construction of a two-dimensional CFT is that each Riemann surface $\Sigma$ with one (positively oriented) boundary circle defines a point $W$ in $\mathrm{Gr}$ by taking $W$ to be the space of $L^{2}$ holomorphic sections of $\kappa^{1 / 2}$ on $\Sigma$. In similar fashion, if $\Sigma$ has $p$ positively oriented and $q$ negatively oriented boundary components the corresponding construction determines an amplitude

$$
\mathscr{H}^{\otimes p} \otimes \overline{\mathscr{H}}^{\otimes q} \rightarrow \operatorname{Det}_{W}^{*},
$$

where $W$ is now a point in $\operatorname{Gr}\left(H^{\oplus(p+q)}\right)$. Thus we have a candidate for a "projective" two-dimensional CFT, in that the amplitudes take values in a complex line which is not, however, canonically isomorphic to $\mathbb{C}$. This line can also be identified with the determinant line

$$
\operatorname{Det}\left(\bar{\partial}_{\Sigma}\right)
$$

of the Cauchy-Riemann operator $\bar{\partial}_{\Sigma}$ on $\Sigma$ (with coefficients in $\kappa^{1 / 2}$ and defined with respect to the standard boundary conditions determined by the parametrization of $b \Sigma$ ).

Now (1.12) behaves well under (1.1) and (1.2); there are canonical isomorphisms

$$
\operatorname{Det}\left(\bar{\partial}_{\Sigma_{1} \amalg \Sigma_{2}}\right)=\operatorname{Det}\left(\bar{\partial}_{\Sigma_{1}}\right) \otimes \operatorname{Det}\left(\bar{\partial}_{\Sigma_{2}}\right)
$$




$$
\operatorname{Det}\left(\bar{\partial}_{\Sigma}\right)=\operatorname{Det}\left(\bar{\partial}_{\Sigma^{\prime}}\right)
$$

and, as explained in [23], (1.5) and (1.6) are satisfied with such a definition. We refer also to [25] for a different account of the use of infinite Grassmannians in this context.

In this paper we shall describe a four-dimensional structure of this type: we shall define a four-dimensional CFT as a Hilbert space representation of the operations of disjoint union and contraction on the class of (Riemannian) conformally flat four-manifolds with parametrized boundaries consisting of a disjoint union of round three-spheres. By applying the same piece of abstract infinite-dimensional geometry in this context, the "classical fields" now being four-dimensional spinor fields, we attempt to construct examples. In this we are not entirely successful, for we are unable to prove the analogue of (1.6) although it seems likely to be true (possibly only generically and for certain spins).

An important aspect of our treatment is the use of complex methods, these being introduced through Penrose's twistor theory. The use of twistor theory here permits a purely holomorphic formulation of four-dimensional CFT, in which CFTs based on conformally flat four-manifolds appear by restriction to the "real slice" (for definitions, see Sect. 2). Indeed, four-dimensional CFT (in the sense of this paper) was initially formulated in terms of (not necessarily real) flat twistor spaces, as explained in $[15,24]$; the relation to Riemannian conformally flat geometry was pointed out subsequently by Le Brun.

In fact we introduce, in Sect. 2, two families of generalizations of Riemann surfaces, which give appropriate settings for higher-dimensional CFT in the sense that they are closed under disjoint union and certain contraction operations. The real family consists of the conformally flat $n$-manifolds with boundary a disjoint union of round $(n-1)$-spheres; the complex family contains certain odd-dimensional complex manifolds with boundary.

In Sect. 3, we give some results about classical fields on conformally flat four-manifolds in terms of their twistor description as analytic cohomology classes and prove some technical results needed in Sect. 4. This is where our proposed definition of four-dimensional CFT appears and the basic example considered. The possible failure of the analogue of (1.6) is also considered in the general context of the definition of the determinant line on a complex manifold of dimension $>1$.

In closing this Introduction, I thank Claude Le Brun, Roger Penrose and Graeme Segal for illuminating and stimulating conversations. The work was done while the author was a Junior Research Fellow at Merton College, Oxford.

\section{Conformal and Complex Geometry}

The Riemann surfaces of two-dimensional CFT can be viewed either as real or as complex objects. The two viewpoints lead to inequivalent higher-dimensional generalizations. In constructing these generalizations we are strongly guided by the requirement that there should exist some analogue of the sewing or contraction operations mentioned in the Introduction. The motivation for these generalizations 
came from consideration of the four-dimensional case where the real and complex objects are intimately related via Penrose's twistor theory $[18,17,10,3,4]$.

2.1 Flat Twistor Spaces and Conformally Flat Four-Manifolds. Recall that a complex 3-manifold $Z$ is a twistor space if through each point there is a complex projective line in $Z$ with normal bundle isomorphic to $\mathcal{O}(1) \oplus \mathcal{O}(1)$. The space of these projective lines is then a complex 4-manifold $\mathscr{M}$ (the complex space-time) with a natural self-dual (holomorphic) conformal structure. This is the remarkable non-linear graviton construction of Penrose [17]. Let us call the twistor space $Z$ flat if the associated space-time $\mathscr{M}$ is (locally) conformally flat. It is equivalent that each point of $Z$ should have a neighbourhood biholomorphic to a neighbourhood of a line in $\mathbb{C} P^{3}$.

Contact with Riemannian conformal geometry is made by imposing reality conditions. Thus we say $Z$ is real if it is fibred by a sub-family of the preferred family of projective lines. Such a sub-family forms a 4-manifold $M$ with Riemannian self-dual conformal structure: in particular, $Z$ is a real flat twistor space if $M$ is (locally) conformally flat.

The construction is reversible: given a self-dual $\mathscr{M}$ or $M$ one can obtain $Z$ in a canonical way $[17,3]$. It is worth pointing out that the construction simplifies drastically when $M$ is conformally flat and depends only upon knowing that the twistor space of $S^{4}$ is $\mathbb{C} P^{3}$ and the corresponding group-theoretic fact that

$$
\mathrm{Conf}_{4} \hookrightarrow \mathrm{PSL}_{4}(\mathbb{C}),
$$

where the left-hand side is the group of conformal motions of $S^{4}$. Indeed, if $M$ is (locally) conformally flat, it admits an atlas $\left\{U_{i}\right\}$ for which all changes of coordinates are (restrictions of) motions in $\operatorname{Conf}_{4}$. The twistor space $Z_{i}$ of $U_{i}$ can then be identified with an open set of $\mathbb{C} P^{3}$ and the conformal motion which attaches $U_{i}$ to $U_{j}$ along $U_{i} \cap U_{j}$ induces, via (2.1), a holomorphic map which attaches $Z_{i}$ to $Z_{j}$ along $Z_{i} \cap Z_{j}$.

2.2 Boundary Conditions for Conformally Flat Manifolds. To give a geometric setting for the definition of a four-dimensional CFT we need a class of compact conformally flat 4-manifolds with boundary. This class is to be closed under appropriate operations of sewing manifolds together across a boundary component. This is a very restrictive requirement: in Riemannian geometry, boundaries of dimension $>1$ have intrinsic and extrinsic local invariants, all of which must be compatible for such sewing to make sense. In the context of conformally flat geometry, this problem can be circumvented by insisting that every connected component of the boundary be a round three-sphere. To see that this makes sense we can argue as follows. For later convenience we give the argument for conformally flat manifolds of arbitrary dimension.

The notion of "round" $S^{n-1}$ 's in $S^{n}$ (with its standard conformally flat structure) makes sense: they are the sections of $S^{n}$ by hyperplanes in $\mathbb{R}^{n+1}$ and are permuted by the action of the conformal group $\operatorname{Conf}_{n}$. To transfer this notion to a conformally flat $M$ we can use the developing map. Recall that for any simply connected conformally flat $M(n \geqq 3)$ the developing map $M \rightarrow S^{n}$ (see, for example, [22]) is a well-defined conformal local diffeomorphism and that it is unique up to 
composition with elements of $\operatorname{Conf}_{n}$. Now if $N \subset M$ is a closed (n-1)-manifold of $M$ with the topological type of $S^{n-1}$, an appropriate tubular neighbourhood $T$ of $N$ in $M$ will be simply connected and hence developable to $S^{n}$. It is natural to call $N$ a round $S^{n-1}$ in $M$ if and only if it goes over diffeomorphically to a round $S^{n-1}$ in $S^{n}$ under development. (The definition makes sense because the developing map is unique up to composition with elements of Conf $_{n}$.)

Let $M$ be a compact oriented conformally flat $n$-manifold with boundary $\partial M$. We shall say that $\partial M$ is of standard type (or, more simply, standard) if it is a disjoint union of round $S^{n-1}$ 's. The above discussion implies that for each boundary component $C$ one can find a neighbourhood of $C$ in $M$ and a conformal diffeomorphism of this neighbourhood onto a neighbourhood of the equator in the northern hemisphere of $S^{n}$. It follows that if we have two boundary components $C$ and $C^{\prime}$ of $M$ and an orientation reversing conformal diffeomorphism $\psi: C \rightarrow C^{\prime}$, then $C$ and $C^{\prime}$ can be attached using $\psi$ and that the resulting manifold will have a unique conformally flat structure compatible with the original one on $M$. This is the crucial observation which motivates the introduction of this class of manifolds as appropriate for the definition of higher dimensional CFT.

Any two allowable attaching maps differ by an element of $\operatorname{Conf}_{n-1}$. When $n>2$, this is a finite-dimensional group, identifiable with $P S O_{n, 1}$ which has real dimension $n(n+1) / 2$. In contrast, when $n=2$, as is well known, the allowable attaching maps are all orientation-reversing diffeomorphisms of the circle.

2.3 Boundary Conditions for Flat Twistor Spaces. Let us now give the twistor description of the conformally flat 4-manifolds with boundary of standard type. The main point is the twistor description of a round $S^{3}$; this is most easily given for a round $S^{3}$ in $S^{4}$ by identifying the latter with the quaternionic projective line $[3,4]$. In homogeneous quaternionic coordinates $\left(q_{1}, q_{2}\right)\left(q_{i} \in \mathbb{H}\right)$, a round $S^{3}$ is given by the homogeneous equation

$$
q_{1} \tilde{q}_{1}-q_{2} \tilde{q}_{2}=0
$$

$(\tilde{a}=$ quaternionic conjugate of $a$ ). We obtain the corresponding surface in twistor space from the complex form of (2.2),

$$
\left|z_{1}\right|^{2}+\left|z_{2}\right|^{2}-\left|z_{3}\right|^{2}-\left|z_{4}\right|^{2}=0 \text {. }
$$

Let us call this real hypersurface $P_{0}$. It divides $\mathbb{C} P^{3}$ into two connected pieces whose closures are

$$
P_{ \pm}=\left\{z: \pm\left(\left|z_{1}\right|^{2}+\left|z_{2}\right|^{2}-\left|z_{3}\right|^{2}-\left|z_{4}\right|^{2}\right) \geqq 0\right\}
$$

which we shall consider in some detail in the next section.

From the above, it is clear that the twistor space of a conformally flat 4-manifold with boundary of standard type will be a real flat twistor space $Z$ with boundary consisting of a disjoint union of copies of $P_{0}$. The terms "copies" in the last sentence has a natural meaning since $P_{0}$, as a real hypersurface in a complex manifold, is an example of what is called a "CR-" or "partially complex" manifold [12] and there is a standard notion of CR-equivalence. (This is (much) stronger than diffeomorphism.) 
Let us say that the (not necessarily real) flat twistor space $Z$ has boundary $b Z$ of standard type if $b Z$ is a disjoint union of copies of $P_{0}$. (By a "real" twistor space with boundary of standard type, we mean the twistor space of a conformally flat manifold $M$ with boundary of standard type. The point is that the fibration of $Z$ by real projective lines must extend up to $b Z$.) The rigidity of CR-manifolds implies that for each component $C$ of a standard boundary, one can find a neighbourhood of $C$ in $Z$ which is biholomorphic to a neighbourhood of $P_{0}$ in $P_{+}$(or $P_{-}$). Thus the class of flat twistor spaces with standard boundary is closed under attaching boundary components by orientation reversing CR-equivalences. Any two such equivalences differ by an orientation preserving CR-equivalence of $P_{0}$. The latter is $P S U_{2,2}$ and hence of real dimension 15 (cf. [18]). Notice that in the real case this agrees with the desciption of the allowable attaching maps at the end of Sect. 2.2, for the condition of preservation of the real lines in $P_{0}$ restricts one to the subgroup $P S L_{2}(\mathbb{H}) \cap P S U_{2,2}$ and this is indeed isomorphic to $\mathrm{Conf}_{3}$.

2.4 Higher-Dimensional Flat Twistor Spaces. One can introduce an analogous class of manifolds modelled on any odd-dimensional complex projective space $\mathbb{C} P^{2 k+1}$. As a provisional piece of notation, let us call a complex $(2 k+1)$-manifold $X$ a flat $(2 k+1)$-twistor space if each point has a neighbourhood biholomorphic to a neighbourhood of a $\mathbb{C} P^{k}$ in $\mathbb{C} P^{2 k+1}$. We say that $X$ has a standard boundary $b X$ if the latter is a union of copies of the CR-manifold defined by

$$
\left|z_{0}\right|^{2}+\cdots+\left|z_{k}\right|^{2}-\left|z_{k+1}\right|^{2}-\cdots-\left|z_{2 k+1}\right|^{2}=0
$$

in $\mathbb{C} P^{2 k+1}$.

The class of flat $(2 k+1)$-twistor spaces with standard boundary is closed under the operation of attaching boundary components by means of orientation reversing CR-equivalences. Any two such equivalences again differ by an element of a finite-dimensional group, this time $P S U_{k+1, k+1}$.

2.5 Summary and Examples. In this section we have introduced for each $n$ the class $\mathscr{C}_{n}$ of compact, oriented conformally flat $n$-manifolds with standard boundary and for each $k$ the class $\mathscr{F}_{2 k+1}$ of compact flat $(2 k+1)$-twistor spaces with standard boundary. These classes are closed under certain natural attaching operations and are therefore appropriate starting points for generalizations of Segal's axiomatization [23] of two-dimensional CFT.

For small $n$ and $k$, the classes are related: $\mathscr{C}_{2}$ and $\mathscr{F}_{1}$ coincide with the class of compact Riemann surfaces with boundary a disjoint union of circles; $\mathscr{C}_{4}$ may be regarded as a subclass of $\mathscr{F}_{3}$, obtained by mapping a manifold to its twistor space. It is appropriate to regard $\mathscr{C}_{4}$ as a real slice of $\mathscr{F}_{3}$, as the examples below indicate.

Example 1. The $n$-sphere with $g$ handles, $S_{g}^{n}$, or the connected sum of $g$ copies of $S^{n-1} \times S^{1}$. To exhibit $S^{n-1} \times S^{1}$ as a conformally flat manifold we note that it is a quotient $S^{n}-\{p, q\} / \Gamma$ of the twice-punctured $n$-sphere by the infinite cyclic group $\Gamma$ generated by a single hyperbolic element (for example, a dilatation) of Conf ${ }_{n}$. Similarly $S_{g}^{n}$ is the quotient of an open set $U$ of $S^{n}$ by $\Gamma=\left\langle\gamma_{1}, \ldots, \gamma_{g}\right\rangle$, where the $\gamma_{i}$ are appropriately chosen hyperbolic elements. A more intuitive description is 
as follows: choose $2 g$ non-intersecting round $S^{n-1}$ 's, $S_{1}, \ldots, S_{2 g}$ in $S^{n}$ and choose $\gamma_{i}$ so as to identify $S_{i}$ with $S_{g+i}$. The result of making the identifications is the $n$-sphere with $g$ handles.

The twistor space $Z_{g}$ of $S_{g}^{4}$ has a highly analogous description: replace the round $S^{3}$ 's above by copies of $P_{0}$ and the $\gamma$ 's by the corresponding projective motions in $P S L_{2}(\mathbb{H})$. However, one can deform this construction to yield non-real flat twistor spaces, simply by deforming the projective motions so that they no longer lie in $P S L_{2}(\mathbb{H})$. For more on this construction we refer to [19].

It is clear how to generalize this construction to obtain examples of the higher-dimensional twistor spaces of Sect. 2.4.

Example 2. The torus. Since the translations of $\mathbb{R}^{n}$ are also conformal motions, the $n$-torus $\mathbb{R}^{n} / \Lambda$ ( $\Lambda$ a lattice) has a natural conformally flat structure. This too can be complexified (when $n=4$ ) and generalized to give higher dimensional twistor spaces.

Example 3. Hyperbolic $n$-manifolds. Any hemisphere in $S^{n}$ carries a conformally flat metric of constant curvature -1 because it can be identified with hyperbolic $n$-space $H^{n}$. The group of isometries of this metric is $P S O_{1, n}$ and one obtains compact conformally flat $n$-manifolds by taking quotients by appropriate discrete subgroups of this isometry group.

It is worth pointing out that if one considers the class of manifolds obtained by removing a finite number of disjoint balls from $S^{n}$ and performing some identifications of the resulting boundary, one gets not all of $\mathscr{C}_{n}$ (unless $n=2$ ) but only manifolds of the form $S_{g}$ with boundary. From the "physical" point of view, then, these manifolds acquire a special significance.

\section{Classical Fields}

In this section we discuss some aspects of the theory of classical fields on spaces in $\mathscr{C}_{n}$ and $\mathscr{F}_{2 k+1}$. In two-dimensional field theory, the story is a familiar one: the classical fields of various spins are holomorphic sections of the half-integer powers of the canonical bundle.

In four dimensions, one has conformally invariant field equations for each half-integer $s$, conformally invariant versions of the spin-s Dirac equation if $s \neq 0$ and of the Laplace equation when $s=0$. Twistor theory supplies a complex description of fields, the Penrose transform, which identifies the space of spin-s fields with the analytic sheaf cohomology group $H^{1}\left(Z, \kappa^{(1 / 2) s+1 / 2}\right)(\kappa=$ canonical bundle of $Z$ ). (Technical note: for any twistor space $Z, \kappa$ admits a unique square root; existence and choice of a fourth root correspond to existence and choice of spin structure on $\mathscr{M}$ or on $M$ if $Z$ is real). The Penrose transform is well covered in the literature $[10,6,4,13]$ and we shall use it to identify four-dimensional fields and cohomology classes without comment.

3.1. Polarization of Massless Fields and Expansion in Elementary States. The twistor description of massless fields on real compactified Minkowski space $M_{0}$ 
deserves special mention. It is given by an isomorphism of the form

$$
H^{1}\left(P_{0}, \kappa^{(1 / 2) s+1 / 2}\right)=\left\{\text { massless spin-s fields on } M_{0}\right\} \text {. }
$$

Notice that each side of this equation is ambiguous. There are various natural definitions of the left-hand side, the "CR-cohomology of $P_{0}$ with coefficients in $\kappa^{(1 / 2) s+1 / 2}$." These correspond roughly to the various degrees of differentiability that can be imposed on the fields on the right-hand side. (The field equations on $M_{0}$ are of course hyperbolic and in particular admit solutions that are not $C^{\infty}[5]$. It is a strength of the twistor picture that the difference between Euclidean and Lorentzian signatures is one of interpretation rather than substance.)

For a given natural definition of the left-hand side, the polarization (positive/ negative-frequency decomposition) of the space of massless fields corresponds to the isomorphism

$$
H^{1}\left(P_{0}, \kappa^{(1 / 2) s+1 / 2}\right)=H^{1}\left(P_{+}, \kappa^{(1 / 2) s+1 / 2}\right) \oplus H^{1}\left(P_{-}, \kappa^{(1 / 2) s+1 / 2}\right)
$$

induced by the Mayer-Vietoris exact sequence of the cover $\left(P_{+}, P_{-}\right)$of $\mathbb{C} P^{3}$. This appealing geometrical description is strongly analogous to the standard polarization of $L^{2}\left(S^{1}\right)$ in two-dimensional field theory. There is also a natural analogue of Laurent series expansion, the expansion of cohomology in elementary states [11]. In a Čech description of cohomology, these are represented by monomials of the form

$$
z_{1}^{i} z_{2}^{j} z_{3}^{k} z_{4}^{l}(i+j+k+l=-2 s-2)
$$

where either $i, j \geqq 0$ and $k, l<0$ (for elementary states based on the line $z_{3}=z_{4}=0$ ) or $i, j<0$ and $k, l \geqq 0$ (for elementary states based on the line $z_{1}=z_{2}=0$ ). These cohomology classes correspond to polynomial fields in $M_{0}$ (relative to appropriate trivializations of the bundles involved). It is a theorem of [11] that there are natural topologies for which this family of cohomology classes is dense in $H^{1}\left(P_{0}, \kappa^{(1 / 2) s+1 / 2}\right)$, those based on $z_{3}=z_{4}=0$ being dense in $H^{1}\left(P_{-}, \kappa^{(1 / 2) s+1 / 2}\right)$ and those based on $z_{1}=z_{2}=0$ being dense in $H^{1}\left(P_{+}, \kappa^{(1 / 2) s+1 / 2}\right)$. For our later purposes, we need the following alternative description, which we give only for states based on $z_{1}=z_{2}=0$.

On a compact complex manifold with boundary such as $P_{-}$, it is natural to try to represent the analytic cohomology by harmonic forms relative to some Hermitian metric. This leads to the $\bar{\partial}$-Neumann problem which is the subject of [12]. The nature of the solution to this problem depends critically on the signature of the Levi form of the boundary, in this case $P_{0}$. Since the Levi form of $P_{0}$ is of type $(+,-)$, it follows from [12, Theorems 3.1.14, 3.1.19, 3.2.10] that one has a strong Hodge decomposition for the space of square integrable $(0, p)$ forms on $P_{-}$ (with coefficients in any holomorphic bundle) and that the space of harmonic $(0, p)$-forms is finite-dimensional if $p \neq 1$.

For the bundle $\kappa^{(1 / 2) s+1 / 2}$ one can write down an infinite sequence of $(0,1)$-forms that are harmonic on $P_{-}$(and which satisfy the $\bar{\partial}$-Neumann boundary conditions) relative to the Fubini-Study metric on $\mathbb{C} P^{3}$ induced by

$$
\left|z_{1}\right|^{2}+\left|z_{2}\right|^{2}+\left|z_{3}\right|^{2}+\left|z_{4}\right|^{2} \text {. }
$$


These are of the form

$$
\frac{p_{n}\left(z_{3}, z_{4}\right) p_{n+2 s}\left(\bar{z}_{1}, \bar{z}_{2}\right)}{\left(\left|z_{1}\right|^{2}+\left|z_{2}\right|^{2}\right)^{n+2 s+2}}\left(\bar{z}_{1} d \bar{z}_{2}-\bar{z}_{2} d \bar{z}_{1}\right)
$$

if $s \geqq 0$ and

$$
\frac{p_{n-2 s}\left(z_{3}, z_{4}\right) p_{n}\left(\bar{z}_{1}, \bar{z}_{2}\right)}{\left(\left|z_{1}\right|^{2}+\left|z_{2}\right|^{2}\right)^{n+2}}\left(\bar{z}_{1} d \bar{z}_{2}-\bar{z}_{2} d \bar{z}_{1}\right)
$$

if $s \leqq 0$ where $p_{i}$ denotes a polynomial in two variables, homogeneous of degree $i$, and $n$ is any non-negative integer, the order of the elementary state.

One can show (by computing the fields they represent, for example) that these are the harmonic representatives for the elementary states based on $z_{1}=z_{2}=0$. Then a straightforward modification of the arguments in [11] yields the following, which we shall need later.

Proposition 3.1. Let $V$ be a neighbourhood of $P_{0}$ in $P_{-}$so small that the line $z_{1}=z_{2}=0$ does not meet it. Let $\alpha$ be a $(0,1)$-form $\alpha$ on $V$ with coefficients in $\kappa^{(1 / 2) s+1 / 2}$ which satisfies

$$
\bar{\partial} \alpha=0, \quad \vartheta \alpha=0
$$

and the $\bar{\partial}$-Neumann conditions at $P_{0}$ (all relative to the Fubini-Study metric as above). Then $\alpha$ has a unique expansion in $V$ as a convergent sum of harmonic elementary states based on $z_{1}=z_{2}=0$ and $z_{3}=z_{4}=0$.

In the above we have used the standard notation $\vartheta$ for the formal adjoint of $\bar{\partial}$.

3.2. Complex Analysis on a Flat Twistor Space with Standard Boundary. Let $Z$ be a connected compact flat twistor space with boundary $b Z$ of standard type. The signature of the Levi form of $b Z$ is the same as that of $P_{0}$, namely $(+,-)$. It follows as in the above discussion for $P_{-}$, that the analytic cohomology of $Z$ is finite dimensional outside degree 1.

The construction of Sect. 4 depends on some properties of the restriction map

$$
i^{*}: H^{1}\left(Z, \kappa^{(1 / 2) s+1 / 2}\right) \rightarrow H^{1}\left(b Z, \kappa^{(1 / 2) s+1 / 2}\right)
$$

induced by the inclusion $i: b Z \rightarrow Z$. We shall usually think of the left-hand side as the space of $L^{2}$ harmonic $(0,1)$ forms on $Z$ relative to some Hermitian metric and the right-hand side accordingly as the $L^{2}$ version of the CR-cohomology of $b Z$. Both sides are actually invariants of the complex manifold $Z$ and its boundary; the choice of Hermitian metric merely moves representatives in their cohomology class.

Theorem 3.2. $i^{*}$ is injective.

Proof. Choose a boundary component $C$ of $b Z$; as we have already noted, there is a neighbourhood $U$ of $C$ in $Z$ which is biholomorphic to a neighbourhood $V$ of $P_{0}$ in $P_{-}$. Let us choose the Hermitian metric on $Z$ so that when transferred to $V$ it becomes the Fubini-Study metric as in Sect. 3.1. If $\alpha \in H^{1}\left(Z, \kappa^{(1 / 2) s+1 / 2}\right)$ then 
when transferred to $V$ it becomes a form satisfying the hypotheses of Proposition 3.1 and so admits a unique expansion in harmonic elementary states (based on a line in $P_{+}$and a line in $\left.P_{-}-V\right)$. Since such a combination of elementary states has vanishing restriction to $P_{0}$ if and only if it vanishes identically in $V, i^{*}(\alpha)=0$ implies $\alpha \mid U=0$. By unique analytic continuation of harmonic forms [2], it follows that $\alpha=0$ in $Z$.

Remarks. 1. Our proof obviously establishes the slightly stronger result that the restriction map to any one boundary component is injective.

2. When $Z$ and $b Z$ are real, the statement has an interpretation in terms of an elliptic boundary value problem on $M$.

3. The expansion theorem for cohomology classes in elementary states is a crucial ingredient in the proof; the result fails in general for cohomology of degree 2 , for example.

Because of this result, we can identify $H^{1}\left(Z, \kappa^{(1 / 2) s+1 / 2}\right)$ with a closed linear subspace of $H^{1}\left(b Z, \kappa^{(1 / 2) s+1 / 2}\right)$. If $\psi: P_{0} \rightarrow C$ is a parametrization of the boundary component $C$ of $b Z$, then by composing with $\psi^{*}$, we can regard $H^{1}\left(Z, \kappa^{(1 / 2) s+1 / 2}\right)$ as a closed linear subspace of $H^{1}\left(P_{0}, \kappa^{(1 / 2) s+1 / 2}\right)$ (depending upon $\psi$ ). (Remark: for non-integral $s$, one needs to choose a bundle isomorphism $\bar{\psi}: \kappa^{(1 / 2) s+1 / 2} \mid P_{0} \rightarrow$ $\kappa^{(1 / 2) s+1 / 2} \mid C$ which covers $\psi$. For ease of exposition, we assume this given whenever needed.) We now come to the key technical result concerning this subspace:

Theorem 3.3. Suppose $\psi: P_{0} \rightarrow C$ extends to a biholomorphic map from a neighbourhood $V$ of $P_{0}$ in $P_{-}$to a neighbourhood $U$ of $C$ in $Z$. Then the orthogonal projection from the subspace $W=H^{1}\left(Z, \kappa^{(1 / 2) s+1 / 2}\right)$ of $H^{1}\left(P_{0}, \kappa^{(1 / 2) s+1 / 2}\right)$ to $H_{+}=H^{1}\left(P_{+}, \kappa^{(1 / 2) s+1 / 2}\right)$ is trace-class.

The proofs of this theorem and the next are the four-dimensional analogues of the proof of Theorem 8.11 .10 of [20].

Proof. Consider the projective motion given in our standard coordinates by

$$
f_{r}:\left(z_{1}, z_{2}, z_{3}, z_{4}\right) \mapsto\left(z_{1}, z_{2}, r z_{3}, r z_{4}\right) .
$$

Choose $r>1$ so small that $f_{r}$ carries $P_{0}$ onto a hypersurface $P_{r}$ in the interior of $V$. Then our projection

$$
W \rightarrow H_{+}
$$

factorizes through restriction to $P_{r}$ and pull-back $f_{r^{-1}}^{*}: H_{+} \rightarrow H_{+}$. To see that this latter map, and hence the orthogonal projection in question, is trace class, we consider its action on the harmonic elementary states based on $z_{1}=z_{2}=0$. Now this action is given by multiplication by $r^{-n}(s \geqq 0)$ and by multiplication by $r^{2 s-n}$ $(s \leqq 0)$ on the subspace of elementary states of order $n$. The dimension of this subspace is the product of the dimension of the space of homogeneous polynomials in two variables of degree $n$ with the dimension of the space of homogeneous polynomials of degree $n+2|s|$ and is thus $(n+1)(n+2|s|+1)$. It follows that the 
trace of the operator $f_{r^{-1}}^{*}$ on $H_{+}$is bounded by a multiple of

$$
\sum_{n=1}^{\infty}(n+1)(n+2|s|+1) r^{-n}<\infty \text {. }
$$

This observation completes the proof.

It is now convenient and suggestive to introduce the following notation. We call a parametrization $\psi$ of a boundary component of $Z$ "in-going" if it extends to an identification of a neighbourhood of $P_{0}$ in $P_{+}$with a neighbourhood of $C$ in $Z$. In the opposite case (the hypothesis of Theorem 3.3) it is called "out-going." For positive integers $l, m$ the polarized Hilbert space $H^{l, m}$ is the $(l+m)$-fold direct sum of the Hilbert space $H$ of $L^{2}$ spin-s massless fields on $M_{0}$ with the polarization

$$
H_{ \pm}^{l, m}=H_{ \pm}^{\oplus l} \oplus H_{+}^{\oplus m} \text {. }
$$

As a corollary of Theorem 3.3 we now give:

Theorem 3. Let $(Z, j)$ be a compact flat twistor space with standard boundary together with a parametrization of $b Z$ so that the first l components are in-going and the remaining $m$ components are out-going. Then $H^{1}\left(Z, \kappa^{(1 / 2) s+1 / 2}\right)$, regarded as a linear subspace of $H^{l, m}$, defines a point of the restricted Grassmannian $\operatorname{Gr}\left(H^{l, m}\right)$.

Remarks. 1. Recall (cf. Sect. 1) the definition of the restricted Grassmannian of a polarized Hilbert space $H_{+} \oplus H_{1}$ as the set of closed linear subspaces $W$ for which the orthogonal projections $\mathrm{pr}_{ \pm}: W \rightarrow H_{ \pm}$are respectively Fredholm and trace-class.

The analogous result in two dimensions is at the heart of the so-called Krichever construction and plays a key role in two-dimensional CFT. Attempts to introduce restricted Grassmannians in the context of higher dimensional QFT have resulted in larger Grassmannians for which $\mathrm{pr}_{-}$is in some Schatten class $L^{p}$ with $p>2$ [16]. From this point of view, the appearance here of a standard restricted Grassmannian is rather surprising.

2. We are going to use methods of complex analysis to establish this result. In the real case, it can be established by the use of elliptic boundary value theory as has been pointed out by Graeme Segal and John Roe (private communication).

3. Precisely similar results hold for the families $\mathscr{F}_{2 k+1}$ and $\mathscr{C}_{n}$. The relevant degree of cohomology for $\mathscr{F}_{2 k+1}$ is $k$.

Proof. By Theorem 3.3, $\mathrm{pr}_{-}$is trace-class; it follows that the image of $\mathrm{pr}_{+}$is closed.

Let $\hat{Z}$ be the compact flat twistor space without boundary which is obtained from $Z$ by attaching (using $j$ ) one copy of $P_{+}$for each in-going boundary component and one copy of $P$ - for each out-going boundary component. The Mayer-Vietoris exact sequence for the cover $(Z, \hat{Z}-$ int $Z)$ of $\hat{Z}$ yields that the map

$$
H^{1}\left(Z, \kappa^{(1 / 2) s+1 / 2}\right) \oplus\left(H_{-}^{l, m}\right)_{\mathrm{an}} \rightarrow\left(H_{+}^{l, m}\right)_{\mathrm{an}} \oplus\left(H_{-}^{l, m}\right)_{\mathrm{an}}
$$

is Fredholm ( $\hat{Z}$ has finite analytic cohomology in all degrees [8]). In this equation the subscripts indicate that we are working with the definition of cohomology in which all forms are real-analytic up to the boundary. This map is a compact perturbation of the map $\mathrm{pr}_{+} \oplus 1$ (the " $\oplus$ " is relative to the above direct sum 
decomposition) so this map and hence this restriction of $\mathrm{pr}_{+}$to the subspaces of analytic elements is also Fredholm. Since we have already established that $\mathrm{pr}_{+}$ has closed image, it follows that it too is Freedholm.

\section{Construction of Four Dimensional Twistor Conformal Field Theories}

Following Segal's definition (described in the Introduction), we wish to define a four-dimensional conformal field theory to be a "Hilbert space representation" of the operations of disjoint union and contraction on the class $\mathscr{C}_{4}$ or $\mathscr{F}_{3}$ (recall the definitions in Sect. 2.5). Because $\mathscr{C}_{4}$ is a real slice in $\mathscr{F}_{3}$, we shall concentrate on the latter. Then it is natural to define a four-dimensional CFT as a functor $\rho$ such that

$$
\rho\left(P_{0}\right)=\mathscr{H}
$$

for some Hilbert space $\mathscr{H}$ and such that for each flat twistor space $(Z, j)$ with boundary parametrized by $j$ so that the first $l$ boundary components are in-going and the remaining $m$ are out-going,

$$
\rho(Z, j): \mathscr{H}^{\otimes l} \otimes \mathscr{H}^{\otimes m} \rightarrow \mathbb{C}
$$

is a multilinear trace-class functional which has the following properties:

$$
\rho\left(\left(Z_{1}, j_{1}\right) \amalg\left(Z_{2}, j_{2}\right)\right)=\rho\left(Z_{1}, j_{1}\right) \otimes \rho\left(Z_{2}, j_{2}\right),
$$

and

$$
\rho\left(Z^{\prime}, j^{\prime}\right)=\operatorname{trace} \rho(Z, j): \mathscr{H}^{\otimes(l-1)} \otimes \overline{\mathscr{H}}^{\otimes(m-1)} \rightarrow \mathbb{C}
$$

whenever $Z^{\prime}$ is obtained from $Z$ by attaching an in-going and an out-going boundary component.

In the next subsection we shall construct a projective representation for which we have not been able to prove (4.4). That is, we define, for each $(Z, j) \in \mathscr{F}_{3}$, a complex line $\mathbf{L}[Z, j]$ and a $\rho$ for which

$$
\rho(Z, j): \mathscr{H}^{\otimes l} \otimes \overline{\mathscr{H}}^{\otimes m} \rightarrow \mathbf{L}[Z, j]
$$

satisfying the compatibility condition for (4.3),

$$
\mathbf{L}\left[Z_{1} \amalg Z_{2}, j \amalg j_{2}\right]=\mathbf{L}\left[Z_{1}, j_{1}\right] \otimes \mathbf{L}\left[Z_{2}, j_{2}\right] \text { (canonical isomorphism). }
$$

While it is plausible that the compatibility condition

$$
\mathbf{L}\left[Z^{\prime}, j^{\prime}\right]=\mathbf{L}[Z, j]
$$

is true in many cases, this, unfortunately, remains unproved.

Section 4.2 is devoted to a discussion of the determinant line $\operatorname{Det}(Z)$ of a complex $n$-manifold $Z$; when applied to a flat twistor space, this behaves well with respect to the operations of disjoint union and contraction. Then the discrepancy between Det and $\mathbf{L}$ gives an alternative way of looking at the potential failure of (4.7).

4.2. Construction of $\rho$ and $\mathbf{L}$. In this subsection, $Z$ will be a compact flat twistor space with standard boundary parametrized so as to have precisely $l$ in-going and $m$ out-going components, $s$ will be a fixed half-integer. We shall write $H_{Z}$ for the 
space of elements of $H^{1}\left(Z, \kappa^{(1 / 2) s+1 / 2}\right)$ whose boundary values lie in the $L^{2}$ CR-cohomology of the boundary.

By Theorem 3.4 we can regard $H_{Z}$ as a point in $\operatorname{Gr}\left(H^{l, m}\right)$, the "trace-class" restricted Grassmannian defined by the polarization (3.6).

Thus the standard infinite-dimensional geometry of Hilbert-space Grassmannians can now be used as in Sect. 1 and [23] to construct $\rho$ and $\mathbf{L}$ which satisfy (4.1), (4.3), (4.5) and (4.6). Indeed, we set

$$
\begin{aligned}
\mathbf{L}[Z, j] & =\operatorname{Det}_{H_{z}}^{*} \\
& =\operatorname{Det}\left(\mathrm{pr}_{+}: H_{Z} \rightarrow H_{+}\right),
\end{aligned}
$$

where "Det" stands for the fibre of the determinant line bundle on Gr in (4.8) and for the determinant line of the Fredholm operator $\mathrm{pr}_{+}$in (4.9). Following the outline in Sect. 1 (cf. (1.10)) we now set

$$
\rho\left(P_{0}\right)=\mathscr{H}=\hat{\Lambda}\left(H_{+} \oplus \bar{H}_{-}\right)
$$

$\left(H_{ \pm}\right.$are given by the right-hand side of (3.2)) and

$$
\rho(Z, j)=\operatorname{eval}_{H_{Z}}
$$

to define our four-dimensional CFT. Indeed, it is not hard to see that the properties (4.3) and (4.6) hold in this case.

To discuss (4.4) and (4.7), note that a contraction operation induces a Mayer-Vietoris type exact sequence

$$
\rightarrow H^{i}\left(Z^{\prime}\right) \rightarrow H^{i}(Z) \rightarrow H^{i}\left(P_{0}\right) \rightarrow
$$

(the notational dependence upon $\kappa^{(1 / 2) s+1 / 2}$ having been suppressed). This gives an exact sequence of the form

$$
A \rightarrow H^{1}\left(Z^{\prime}\right) \rightarrow H^{1}(Z) \rightarrow H^{1}\left(P_{0}\right) \rightarrow B,
$$

where $A$ is contained in $H^{0}\left(P_{0}\right)$ and $B$ is contained in $H^{2}\left(Z^{\prime}\right)$.

On the other hand it is shown in [23] that a diagram of the form

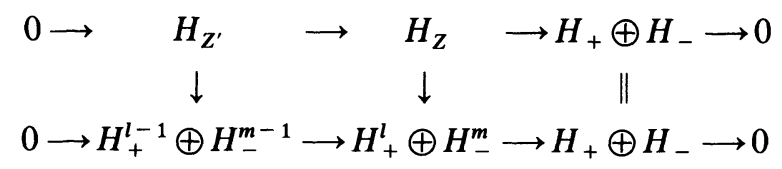

with exact rows, and commuting modulo operators of trace class, implies the remaining conditions (4.4) and (4.7) for $\rho$ and $\mathbf{L}$. So if $A$ and $B$ are zero, these compatibility conditions hold. We can always arrange $A=0$ by taking $s>-1$. In that case:

Proposition 4.1. Suppose that $s>-1$ and the map

$$
H^{2}\left(Z^{\prime}, \kappa^{(1 / 2) s+1 / 2}\right) \rightarrow H^{2}\left(Z, \kappa^{(1 / 2) s+1 / 2}\right)
$$

is injective. Then $\rho$ and $\mathbf{L}$ are compatible with the contraction operation $Z \rightarrow Z^{\prime}$ in $\mathscr{F}_{3}$ (i.e. (4.4) and (4.7) hold).

Proof. The condition of the Proposition guarantees $B=0$. 
Remarks. 1. It is too much to hope that $H^{2}(Z)=0$ for all flat twistor spaces and all s. For example, if $Z$ is compact and has no boundary, $H^{2}(Z, \kappa) \neq 0$ if $H^{5}(Z, \mathbb{C}) \neq 0$. However it is plausible that $s=0$ and $s= \pm \frac{1}{2}$, this map may be injective for generic $Z_{g}$ with boundary.

2. Similar considerations apply to constructing CFT based on the class $\mathscr{F}_{2 k+1}$, where the relevant degree of cohomology would be $k$.

4.2. Determinant Lines for Flat twistor Spaces. Our purpose here is to define the determinant line of a complex $n$-manifold $Z$ (possibly with boundary $b Z$ ) with values in the holomorphic vector bundle $\mathscr{E}$, in terms of the $\bar{\partial}$ complex

$$
\Omega^{0,0} \otimes \mathscr{E} \rightarrow \Omega^{0,1} \otimes \mathscr{E} \rightarrow \cdots .
$$

If $Z$ is compact, the natural definition is

$$
\begin{aligned}
\operatorname{Det}(Z, \mathscr{E}) & =\operatorname{Det}^{*} H^{\text {even }}(Z, \mathscr{E}) \otimes \operatorname{Det} H^{\text {odd }}(Z, \mathscr{E}) \\
& =\operatorname{Det}^{*} H^{0} \otimes \operatorname{Det} H^{1} \otimes \operatorname{Det}^{*} H^{2} \otimes \cdots
\end{aligned}
$$

Here Det $V$ is just the top exterior power of $V$ (for $V$ a finite-dimensional vector space), Det ${ }^{*} V$ is dual. The general properties of such determinant lines have recently been analyzed in a series of papers [7]; they have also been used in the study of self-dual Yang-Mills theory in [9].

We are more interested, however, in a notion of Det for complex manifolds with boundary; for this we need to introduce boundary conditions since $H^{*}(Z, \mathscr{E})$ is not usually finite dimensional. We write such boundary conditions in terms of a Fredholm map of complexes

$$
H^{*}(Z, \mathscr{E}) \rightarrow H_{+}^{*}(b Z, \mathscr{E}),
$$

where the choice of the right-hand side will depend on the particular geometric situation being studied. Then it makes sense to set

$$
\operatorname{Det}(Z, \mathscr{E})=\operatorname{Det}\left(H^{\text {even }}(Z, \mathscr{E}) \rightarrow H_{+}^{\text {even }}(b Z, \mathscr{E})\right) \otimes \operatorname{Det}^{*}\left(H^{\text {odd }}(Z, \mathscr{E}) \rightarrow H_{+}^{\text {odd }}(b Z, \mathscr{E})\right)
$$

This reduces to (4.14) when $b Z=\varnothing$ and $H_{+}^{*}=0$.

Of course both (4.14) and (4.17) reduce to the standard definitions [21,23] when $n=1$ and $Z$ is a Riemann surface. If, in the general case, $b Z$ has Levi form of signature $(p, n-p-1)$, then by standard finiteness theorems $[1,12]$ one can take

$$
H_{+}^{i}(b Z, \mathscr{E})=0 \text { for } i \neq n-p-1 .
$$

In particular, we may use (4.17) and (4.18) to define the determinant line of a compact flat twistor space with boundary. The boundary conditions required when $i=1$ are determined by the parametrization; reverting to the notation introduced at the beginning of Sect. 4.1 we put

$$
H_{+}^{1}(b Z)=H_{+}^{\text {an }}
$$

and write the resulting determinant line as $\operatorname{Det}(Z, s, j)$.

It should come as no surprise to the reader that the obstruction to having 
$\mathbf{L}[Z, j]=\mathbf{L}\left[Z^{\prime}, j^{\prime}\right]$ has been incorporated into the definition of $\operatorname{Det}(Z, s, j)$ so that

$$
\operatorname{Det}(Z, s, j)=\operatorname{Det}\left(Z^{\prime}, s, j^{\prime}\right) \text {. }
$$

In fact, we have

$$
\operatorname{Det}(Z, s, j)=\mathbf{L}[Z, j] \otimes \operatorname{Det}^{*} H^{2}\left(Z, \kappa^{(1 / 2) s+1 / 2}\right),
$$

and (4.20) follows from the exact sequence of Sect. 4.1. From this point of view, we see that Proposition 4.1 can be reformulated as follows.

Proposition 4.2. The conclusion of Proposition 4.1 holds whenever we have a canonical isomorphism

$$
\operatorname{Det} H^{2}\left(Z^{\prime}, \kappa^{(1 / 2) s+1 / 2}\right)=\operatorname{Det} H^{2}\left(Z, \kappa^{(1 / 2) s+1 / 2}\right) .
$$

\section{Discussion}

We have described the construction of a four-dimensional CFT as a natural generalization of the geometric formulation of two-dimensional CFT, emphasizing the links with complex analysis and geometry by the use of twistor theory. Only in Sect. 4.2 did a significant difference begin to emerge; this we related to the discrepancy between what might be termed the "geometric" and the "algebraic" definitions of the determinant line of a flat twistor space. The fact that the conformal group is infinite-dimensional in two dimensions and is finite-dimensional in four dimensions played no significant role in the theory as developed here.

There are number of directions in which one might hope to develop the material we have presented here. In so far as it is a natural generalization of two-dimensional CFT one can ask four-dimensional analogues of all the questions that are interesting in two dimentions (at least those in which the infinite dimensionality of the two-dimensional conformal group plays a subsidiary role). Many of these come down to problems in complex analysis on flat twistor spaces while others relate to questions about "moduli spaces" of flat twistor spaces. Some aspects of the geometry of flat twistor spaces are investigated in a forthcoming paper by M. G. Eastwood and the author. They emerge as an interesting class of complex manifolds - although they are not Kähler [14], it turns out that many natural questions can be answered.

A somewhat more ambitious line of generalization is suggested by the philosophy set out in the monograph by Pressley and Segal [20]: that ideas from (in that case, two-dimensional) quantum field theory may yield a good framework for the study of infinite-dimensional Lie groups (so far, only loop groups and $\operatorname{Diff}\left(S^{1}\right)$. Thus one might hope that some generalization of four-dimensional CFT might yield insights into the groups $\operatorname{Diff}\left(S^{3}\right)$ and $\operatorname{Map}\left(S^{3}, G\right)$ (where $G$ is a compact Lie group). Such a generalization would, perhaps, involve an appropriate formulation of (self-dual) Yang-Mills theory on conformally flat four-manifolds.

A related point is that according to the programme initiated. by Ward and further developed by Mason and Sparling, many two-dimensional integrable models can be understood at the classical level as reductions of the self-dual Yang-Mills equations (and so also in terms of twistor theory). It is thus possible 
that the associated two-dimensional CFT might have some uniform description as reductions of a four-dimensional CFT based on self-dual Yang-Mills theory.

\section{References}

1. Andreotti, A., Grauert, H.: Théorèmes de finitude pour la cohomologie des espaces complexes. Bull. Soc. Math. France 90, 193-259 (1962)

2. Aronszajn, N.: A unique continuation theorem for solutions of elleptic partial differential equations or inequalities of the second order. J. Math. Pures Appl. 36, 235-249 (1957)

3. Atiyah, M. F. Hitchin, N. J. Singer, I.M.: Self-duality in four-dimensional Riemannian geometry. Proc. R. Soc. Lond. A362, 425-461 (1978)

4. Atiyah, M. F.: Geometry of Yang-Mills fields. Lezioni Fermiane, Accademia Nazionale dei Lincei \& Scuola Normale Superiore, Pisca (1979)

5. Bailey, T. N., Ehrenpreis, L., Wells, R. O., Jr.: Weak solutions of the massless field equations. Proc. R. Soc. Lond. A384, 403-425 (1982)

6. Baston, R. J., Eastwood, M. G.: The Penrose transform: Its interaction with representation theory. Oxford Mathematical Monographs, Oxford: O.U.P. 1989

7. Bismut, J.-M., Gillet, H., Soule': Analytic torsion and holomorphic determinant bundles I-III. Commun Math. Phys. 115, 49-78, 79-126 and 301-351 (1988)

8. Cartan, H., Serre, J.-P.: Un théorème de finitude concernant les variétés analytiques compacts. C. R. Acad. Sc. 237, 128-130 (1953)

9. Donaldson, S. K.: Infinite determinants, stable bundles and curvature. Duke Math. J. 54, 231-247 (1987)

10. Eastwood, M. G., Penrose, R., Wells, R. O., Jr.: Cohomology and massless fields. Commun. Math. Phys. 78, 305-351 (1981)

11. Eastwood, M. G., Pilato, A. M.: The density of twistor elementary states. In: Mason, L. J., Hughston, L. P. (eds.). Further advances in twistor theory. Oxford: Pitman 1990

12. Folland, G. B., Kohn, J. J.: The Neumann problem for the Cauchy-Riemann complex. Ann. of Math. Studies vol. 75. Princeton: P.U.P. 1972

13. Hitchin, N. J.: Linear field equations on self-dual spaces. Proc. R. Soc. Lond. A370, 173-191 (1980)

14. Hitchin, N. J.: Kählerian twistor spaces. Proc. Lond. Math. Soc. 43, 133-150 (1981)

15. Hodges, A. P., Penrose, R., Singer, M. A.: A twistor conformal field theory for four space-time dimensions. Phys. Lett. B216, 48-52 (1989)

16. Mickelsson, J., Rajeev, S. G.: Current algebras in $d+1$ dimensions and determinant bundles over infinite-dimensional Grassmannians. Commun. Math. Phys. 116, 365-400 (1988)

17. Penrose, R.: Nonlinear gravitons and curved twistor theory. Gen. Rel. Grav. 7, 31-52, (1976)

18. Penrose, R., Rindler, W.: Spinors and space-time, vol. 2. Cambridge: C.U.P. 1986

19. Penrose, R.: Pretzel twistor spaces. In: Mason, L. J., Hughston, L. P. (eds.). Further advances in twistor theory. Oxford: Pitman 1990

20. Pressley, A. Segal, G. B.: Lomp groups. Oxford Mathematical Monograph. Oxford: O.U.P. 1986

21. Quillen, D. G.: Determinants of Cauchy-Riemann operators over a Riemann surface. Funct. Anal. Appl. 19, 31-4 (English) (1985)

22. Schoen, R., Yau, S.-T.: Conformally flat manifolds, Kleinian groups and scalar curvature. Invent. Math. 92, 47-71 (1988)

23. Segal, G. B.: The definition of conformal field theory (to appear, 1990)

24. Singer, M. A.: Twistors and conformal field theory. In: Mason, L. J. (ed.). Further advances in twistor theory. Oxford: Pitman 1990

25. Witten, E.: Quantum field theory, grassmannians and algebraic curves. Commun. Math. Phys. 113, $529-600$ (1988)

Communicated by A. Connes 